\title{
MENINGOCOCCAL DISEASE IN AN AMBULANCE WORKER
}

\author{
Francesco Maria Fusco (fusco@inmi.it) ${ }^{1}$, Vincenzo Puro ${ }^{1}$ \\ 1. National Institute for Infectious Diseases “Lazzaro Spallanzani”, Rome, Italy
}

To the Editor: We read the interesting communication by Petsas et al. about a secondary case of meningococcal disease in an ambulance worker that was recently published in your journal [1]. In the described case, the worker was not considered as a close contact, and chemoprophylaxis was not offered.

In our opinion, this was wrong for at least two reasons. First of all, the ambulance worker did not use droplet precautions, i.e. he did not wear a surgical mask although this is recommended. Indeed, it is very unlikely that the distance between his face and the patient's head was never less than $90 \mathrm{~cm}$, given that he gave support in moving the patient from a chair, down the stairs and up to the ambulance, always standing at the patient's head holding an oxygen face mask in place.

Moreover, an additional factor that could have increased the risk of transmission was the use of the oxygen face mask during the transport. Experimental studies suggest that oxygen face masks produce turbulent fluxes of aerosols, which could contain potentially infectious droplets $[2,3]$. In one experiment, a subject inhaled saline mist and exhaled through three different models of oxygen masks, in order to illustrate the pattern of dispersal of pulmonary gas. In two commonly used masks, exhaled gas formed a plume emanating from the side vents. In a second study, a human lung model (respiration rate, 12 breaths/min) was designed to test the potential for a simple oxygen mask in a common setting (4 litres/min) to disperse potentially infectious exhaled air into the surrounding area. A laser sheet was used to illuminate the exhaled air from the mask, which contained fine tracer smoke particles. These experimental observations evaluated the distance reached by aerosols produced by a patient correctly wearing an oxygen face mask, and showed that the exhaled air at the peak of the simulated exhalation reached a distance of approximately 40 centimetres.

A potential role of oxygen face masks in the transmission of droplet-transmitted diseases (in particular of SARS and other respiratory diseases prone to cause epidemics) is also considered in some guidelines, in which the use of standard low flow oxygen (oxygen flow rates of under 6 litres/minute) [4], or the addition of an expiratory port with a bacterial/viral filter are recommended [5].

In the described case, the patient was drowsy and agitated, and he repeatedly attempted to remove the oxygen face mask during the journey, probably causing repeated and unpredictable clouds of aerosol containing infectious droplets.

We strongly agree with the authors' conclusion that the correct application of droplet precautions, including the use of surgical masks, should always be observed when caring for a suspected case of meningitis. When oxygen supplementation is needed, further precaution measures, such as the use of standard low flow oxygen or placement of bacterial/viral filters should be considered.

\section{References}

1. Petsas A, Sharma A, Aghadiuno 0, Abid M, Paranthaman K. A secondary case of meningococcal disease in an ambulance worker, Berkshire, November 2007. Euro Surveill. 2008;13(4). Available from: http://www.eurosurveillance.org/ edition/v13n04/080124_1.asp

2. Hui DS, Ip M, Tang JW, Wong AL, Chan MT, Hall SD, et al. Airflows around oxygen masks: A potential source of infection? Chest. 2006;130(3):822-6.

3. Somogyi R, Vesely AE, Azami T, Preiss D, Fisher J, Correia J, et al. Dispersal of respiratory droplets with open vs closed oxygen delivery masks: implications for the transmission of severe acute respiratory syndrome. Chest. 2004;125(3):1155-7.

4. Lim WS, Anderson SR, Read RC; SARS Guidelines Committee of the British Thoracic Society; British Infection Society; Health Protection Agency. Hospital management of adults with severe acute respiratory syndrome (SARS) if SARS re-emerges--updated 10 February 2004. J Infect. 2004;49(1):1-7.

5. World Health Organization. Infection prevention and control of epidemicand pandemic-prone acute respiratory diseases in health care. WHO interim guidelines. June 2007. Available from: http://www.who.int/csr/resources/ publications/WHO_CDS_EPR_2007_6c.pdf

This article was published on 6 March 2008.

Citation style for this article: Meningococcal disease in an ambulance worker. Euro Surveill. 2008;13(10):pii=8061. Available online: http://www.eurosurveillance.org/ ViewArticle.aspx?ArticleId $=8061$ 